\section{Neues Argument für Frühtherapie bei HIV-Infektion}

— Wird bei HIV-Infizierten die antiretrovirale Therapie frühzeitig nach der Infektion begonnen, also noch während des spontanen, aber nur vorübergehenden Anstiegs der CD4-Zellzahl im peripheren Blut, ist die Chance auf eine anhaltende Stärkung der Immunabwehr deutlich größer als bei einem späteren Therapiebeginn (s. auch S. $24 \mathrm{ff}$.).

Bereits Mitte der 1990er-Jahre plädierten viele HIV-Therapeuten für einen frühen Behandlungsbeginn. Doch bisher war nicht klar, wie sich der Zeitpunkt des Therapiebeginns auf das Immunsystem auswirkt. Um dies zu prüfen, wurde in einer prospektiven US-Studie zwischen 1996 und 2010 bei HIVInfizierten die antiretrovirale Therapie in- nerhalb von vier Monaten nach der vermuteten Infektion oder später begonnen. Als primärer Endpunkt wurde ein CD4-Wert von mindestens 900/ $\mu$ l gewählt. Diese Zellzahl kristallisierte sich aus der Blutanalyse bei mehr als 16000 HIV-negativen Teilnehmern in 25 Studien als Mittelwert und als Surrogatmarker für ein starkes Immunsys-

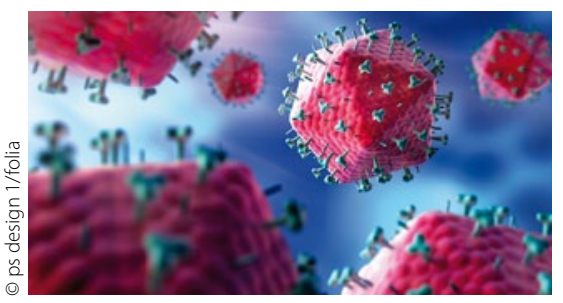

Was hält HI-Viren am besten in Schach?

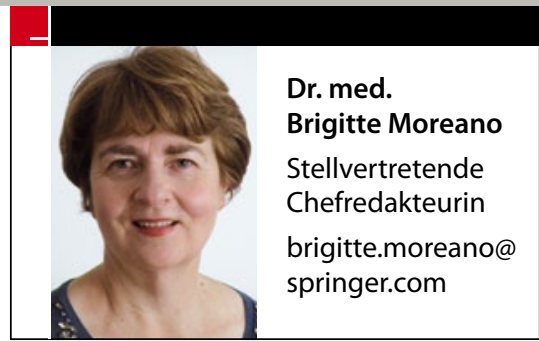

tem heraus. Durch die Frühtherapie erreichten signifikant mehr Patienten (64\%) innerhalb der vier Jahre den primären Endpunkt, dagegen nur 34\% der Patienten, deren HIV-Therapie frühestens vier Monate nach der Infektion begonnen worden war. Unabhängig vom Zeitpunkt des Therapiebeginns war die Wahrscheinlichkeit, den primären Endpunkt zu erreichen, bei jenen HIV-Infizierten drastisch verringert, deren CD4-Wert beim Therapiestart mit weniger als $500 / \mu$ l schon sehr niedrig war.

Le Tet al. N Engl J Med 2013; 368: 218-30; doi: 10.1056/ NEJMoa1110187

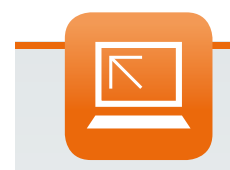

Weitere Infos auf springermedizin.de

\title{
Todesursachen für HIV-Patienten haben sich gewandelt
}

- Dank antiretroviraler Therapien werden Menschen mit HIV-Infektionen immer älter und die allermeisten HIV-Patienten sterben nicht an AIDS.

In der Swiss HIV Cohort Study (SHCS) wurden zwischen 1988 und 2010 die Todesursachen von HIV-infizierten Personen nach ICD-10 registriert. Während in den Jahren bis 1995 noch $78 \%$ der Patienten an AIDS starben, waren es von 1996-2004 nur noch $41 \%$. Zwischen 2005 und 2010 war die Rate auf $15 \%$ gesunken. Als Todesursache sind bei HIV-infizierten Personen maligne Geschehen, Lebererkrankungen, andere Infektionen und kardiovaskuläre Ereignisse immer mehr in den Vordergrund gerückt. Vor allem Koinfektionen mit Hepatitis B und $C$ erhöhen das Sterberisiko.

Nach dem seit 2005 zusätzlich verwendeten Coding Causes of Death in HIV-(CoDe-) Protokoll ergaben sich folgende Ursachen:
19\% AIDS-unabhängige maligne Erkrankungen einschließlich des hepatozellulären Karzinoms (HCC) infolge Hepatitis $B$ oder C, 16\% AIDS, 15\% Leberversagen (ohne HCC), 9\% AIDS-unabhängige Infektionen, 7\% Substanzmissbrauch, $6 \%$ Suizid, $6 \%$ Myokardinfarkt (vgl. S. 32 ff. in diesem Heft).

Diese Entwicklung spiegelt auch eine Untersuchung von Wada et al. aus dem Januar diesen Jahres wider. Hier wurde gezeigt, dass nach Beginn der hoch aktiven antiretroviralen Therapie (HAART) die AIDS-bedingte Mortalität in den USA von $94 \%$ vor 1996 auf etwa 50\% nach 1996 gesunken ist. Deutlich zeichnete sich der Gewinn an Lebenszeit ab: Für HIV-Positive, die an AIDS starben, betrug er fünf Jahre. HIV-Patienten mit anderen Todesursachen lebten 17 Jahre länger als vor 1996.

Weber R. et al. HIV Medicine 2013; 14(4): 195-207

\section{Dossiers und Schwerpunkte zum Thema auf \\ - www.springermedizin.de \\ Dossier Sexualität: 3572634 \\ Dossier HIV \& Aids: 303946 \\ Dossier Geschlechtskrankheiten: \\ - 474118 \\ Schwerpunkt Infektiologie: \\ - 299052 \\ Schwerpunkt Dermatologische \\ Infektionen und Venerologie: \\ > 2336722 \\ Blickdiagnosen AIDS: \\ Drei pathognomonische Opportu- nisten auf einen Blick: 2999420 \\ Steigende Krebssterblichkeit - Bei \\ HIV: ein Auge auf Tabuzonen wer- \\ fen! 2999430 \\ Die Beiträge finden Sie, indem \\ Sie die ID-Nummer in die Suche \\ eingeben.}

